\title{
Increased Risk of Pancreatitis after Endoscopic Retrograde Cholangiopancreatography Following a Positive Intraoperative Cholangiogram: A Single-Center Experience
}

\author{
Lalitha M. Sitaraman', Rita M. Knotts², Judith Kim', Srihari Mahadev² and David S. Lee ${ }^{2}$ \\ ${ }^{1}$ Department of Medicine, ${ }^{2}$ Division of Digestive and Liver Diseases, Columbia University Irving Medical Center, New York, NY, USA
}

Background/Aims: To determine if patients with a positive intraoperative cholangiogram (IOC) who undergo a subsequent endoscopic retrograde cholangiopancreatography (ERCP) have an increased risk of post-ERCP pancreatitis (PEP) compared to those who undergo ERCP directly for suspected common bile duct stones.

Methods: A retrospective case-control study was performed from 2010 to 2016. Cases included inpatients with a positive IOC at cholecystectomy who underwent subsequent ERCP. The control group included age-sex matched cohorts who underwent ERCP for choledocholithiasis. Multivariate logistic regression was used to assess the association between PEP and positive IOC, adjusting for matching variables and additional potential confounders.

Results: Of the 116 patients that met the inclusion criteria, there were 91 women (78\%) in each group. Nine patients (7.8\%) developed PEP in the IOC group, compared to 3 patients in the control group (2.6\%). The use of pancreatic duct stents and rectal indomethacin was similar in both groups. After adjusting for age, sex, total bilirubin levels, and any stent placement, patients with a positive IOC had a significantly increased risk of PEP (odds ratio, 4.79; 95\% confidence interval, $1.05-21.89 ; p<0.05$ ).

Conclusions: In this single-center case-control study, there was a five-fold increased risk of PEP following a positive IOC compared to an age-sex matched cohort. Clin Endosc 2021;54:107-112

Key Words: Cholangiogram; Cholecystectomy; Endoscopic retrograde cholangiopancreatography; Pancreatitis

\section{INTRODUCTION}

Cholelithiasis is very common in the general population, and when symptomatic, cholecystectomy (CCY) is often indicated. Since the development of the intraoperative cholangiogram (IOC), many have called for its routine use in preventing bile duct injury. ${ }^{1,2}$ However, the use of IOC is not limited to

Received: January 20, 2020 Revised: May 9, 2020

Accepted: May 11, 2020

Correspondence: David S. Lee

Division of Digestive and Liver Diseases, Columbia University Irving Medical Center, 161 Fort Washington Ave, Herbert Irving Pavilion, Room 862, New York, NY 10032, USA

Tel: +1-212-342-1864, Fax: +1-212-305-5576, E-mail: Lalitha.Sitaraman@gmail. com

ORCID: https://orcid.org/0000-0002-0116-992X

cc This is an Open Access article distributed under the terms of the Creative Commons Attribution Non-Commercial License (http://creativecommons.org/ licenses/by-nc/3.0) which permits unrestricted non-commercial use, distribution, and reproduction in any medium, provided the original work is properly cited. simply delineating the anatomy of the biliary tree; IOC can also be used to detect and flush stones from the biliary tree. Balloon clearance devices, such as Fogarty balloons, are also recommended for intraoperative stone removal. ${ }^{3}$

When stones are detected and unable to be cleared during IOC, post-operative endoscopic retrograde cholangiopancreatography (ERCP) is required for their removal. ${ }^{4}$ ERCP carries a risk of complications, most commonly bleeding and pancreatitis. ${ }^{5}$ The rate of post-ERCP pancreatitis (PEP) varies in the literature, anywhere from $2.1 \%-24.4 \%{ }^{6}$ Even large cohort studies present diverse results; a recent systematic review of 13,296 patients demonstrated a rate of $9.7 \%$, another with 16,855 patients had a rate of $3.47 \%$, while a third one with 11,497 patients had a rate of $2.6 \% .^{7-9}$

There are many reasons a patient can be at high risk of developing PEP, including but not limited to female gender, normal serum bilirubin, biliary sphincter balloon dilation, difficult cannulation, pancreatic sphincterotomy, and one or more injections of contrast medium into the pancreatic duct 
(PD). ${ }^{10}$ Attempting to clear filling defects during IOC often involves "aggressive" flushing of the duct or multiple attempts of flushing with the use of glucagon. If stones are unable to flow into the duodenum, "aggressive" flushing or the use of balloon clearance devices could potentially increase the risk of stone impaction at the papilla, trauma to the papilla, and inadvertent $\mathrm{PD}$ injection. Consequently, this could also make the subsequent ERCP more difficult, requiring more aggressive techniques for bile duct cannulation and clearance of the duct, therefore increasing the risk of PEP.

The risk of PEP in patients with a positive IOC has not been investigated before. The aim of this study was to determine if there was an increased risk of PEP in patients with a positive IOC, compared to those who had ERCP alone, while controlling for various known risk factors.

\section{MATERIALS AND METHODS}

After approval from the Columbia University Institutional Review Board, a retrospective case-control study was conducted at Columbia University Medical Center from 2010 to 2016. A query was performed to identify all cases of laparoscopic CCY with IOC from 2010 to 2016. Inpatients with a positive IOC-defined as no flow of contrast medium into the duodenum or visible filling defect in the bile duct-who were referred for post-operative ERCP were eligible for inclusion.

Each subject's medical record was reviewed, including consultation notes, progress notes, imaging reports, laboratory data, operative reports, and endoscopy reports, to determine if the patient met the study group criteria. Inpatients who had undergone ERCP for choledocholithiasis without IOC were selected as controls and matched in a 1:1 ratio by age and sex. All patients admitted with gallstone pancreatitis or those with a history of PEP were excluded from both groups. Patients were also excluded if they had prior ERCP.

Patients were defined as having PEP if they developed new onset abdominal pain, elevation in amylase and/or lipase greater than three times the upper limit of normal more than 24 hours after ERCP, and clinical symptoms of pancreatitis within seven days post-ERCP. After excluding patients with elevated amylase or lipase levels on admission, clinical data were collected regarding laboratory values and imaging performed on admission; specifically, the results of serum liver function tests, and ultrasound or magnetic resonance cholangiopancreatography (MRCP) scans. Details on the techniques practiced during IOC, including the use of glucagon, balloon or basket clearance devices, and the report of "aggressive" or "vigorous" flushing, were noted. Information about the ERCP, including stent placement and the use of rectal indomethacin, was also collected.

Statistical analysis was performed using Stata software (StataCorp LLC; College Station, TX, USA). All normally distributed data were analyzed using an unpaired Student's $t$-test. Variables found to be non-normally distributed were analyzed using Mann-Whitney $U$ test. Comparisons of proportions between groups were carried out using chi-square test or Fisher's exact test. After calculating the unadjusted association between PEP and a positive IOC ("Model 1"), multivariable logistic regression was performed to assess the association between PEP and having a positive IOC, adjusting for matching variables and potential confounders. The first multivariable model ("Model 2") included age and sex as the matching variables. We subsequently generated a parsimonious model for the association between PEP and positive IOC ("Model 3"), based on the collected data. Each variable was assessed, one at a time, to determine whether that particular variable was a potential confounder for the association between PEP and positive IOC. Covariates were defined as potential confounders if they altered the $\beta$ coefficient for positive IOC by $>10 \%$, and these were included in an initial full model. Variables with the highest $p$-value and $>0.1$ were then sequentially removed. This process was continued until a final parsimonious model was developed. Statistical significance was defined as $p<0.05$.

\section{RESULTS}

Of the 677 cases with CCY and IOC, 116 patients met the inclusion criteria, and 116 control patients were matched on age and sex. The most common reasons for exclusion were elevated lipase on admission or ERCP performed before CCY. There were 91 women and 25 men in both groups. Baseline characteristics, as available, are shown in Table 1. Patients who underwent ERCP directly without IOC were more likely to have elevated bilirubin levels, elevated alkaline phosphatase levels, and a larger common bile duct. Nearly every patient with a positive IOC had an abdominal ultrasound prior to CCY; those who did not undergo IOC were more likely to have an MRCP.

Information on the use of additional techniques during IOC was collected from the operative reports, including the use of glucagon, "aggressive" or "vigorous" flushing of the duct, and the use of balloon clearance devices (Table 2). Though half of the patients had glucagon injected to attempt to clear the duct, the use of balloon clearance devices was less common (24\%). Documented subjective reports of "aggressive" flushing were low (5\%).

The rates of PEP depending on the IOC techniques are reported in Table 2 and demonstrated in Fig. 1. There was 
Table 1. Baseline Characteristics

\begin{tabular}{lccc}
\hline & Positive IOC $(\boldsymbol{n}=\mathbf{1 1 6})$ & No IOC $(\boldsymbol{n}=\mathbf{1 1 6})$ & $p$-value \\
\hline Age $($ mean \pm SD) & $44.1(19.1)$ & $44.2(18.9)$ & 0.95 \\
Sex, Men, $n(\%)$ & $25(21.5)$ & $25(21.5)$ & 1 \\
Amylase, mean \pm SD $(\mathrm{U} / \mathrm{L})$ & $41.2(18.1)^{\mathrm{a})}$ & $48.4(30.8)^{\mathrm{a})}$ & 0.08 \\
Lipase, mean $\pm \mathrm{SD}(\mathrm{U} / \mathrm{L})$ & $29.1(22.4)^{\mathrm{b})}$ & $27.5(21.4)^{\mathrm{b})}$ & 0.59 \\
Total bilirubin, mean $\pm \mathrm{SD}(\mathrm{mg} / \mathrm{dL})$ & $2.7(2.6)^{\mathrm{c})}$ & $3.8(4.5)^{\mathrm{c})}$ & 0.02 \\
Direct bilirubin, mean $\pm \mathrm{SD}(\mathrm{mg} / \mathrm{dL})$ & $1.5(1.7)^{\mathrm{c})}$ & $2.3(2.9)^{\mathrm{c}}$ & 0.01 \\
AST, mean $\pm \mathrm{SD}(\mathrm{U} / \mathrm{L})$ & $298(306)^{\mathrm{c})}$ & $265(264)^{\mathrm{c}}$ & 0.38 \\
ALT, mean $\pm \mathrm{SD}(\mathrm{U} / \mathrm{L})$ & $299(260)^{\mathrm{c}}$ & $298.6(309)^{\mathrm{c}}$ & 0.98 \\
Alkaline phosphatase, mean $\pm \mathrm{SD}(\mathrm{U} / \mathrm{L})$ & $166(92.7)^{\mathrm{c}}$ & $241.4(201)^{\mathrm{c}}$ & $<0.001$ \\
Abdominal ultrasound performed, $n(\%)$ & $114(98.3)$ & $102(87.9)$ & 0.002 \\
MRCP performed, $n(\%)$ & $25(21.6)$ & $42(36.2)$ & 0.01 \\
Common bile duct size, mean $\pm \mathrm{SD}(\mathrm{mm})$ & $8.6(4.1)^{\mathrm{d})}$ & $<0.001$ \\
\hline
\end{tabular}

ALT, alanine transaminase; AST, aspartate transaminase; IOC, intraoperative cholangiogram; MRCP, magnetic resonance cholangiopancreatography; SD, standard deviation.

a) 68 patients/79, ${ }^{\text {b) }} 106$ patients/108, ${ }^{\text {c) }} 111$ patients/116, ${ }^{\text {d) }} 106$ patients/101.

Table 2. Techniques during Intraoperative Cholangiogram and Rate of Post-Endoscopic Retrograde Cholangiopancreatography Pancreatitis

\begin{tabular}{|c|c|c|c|c|c|c|}
\hline & Glucagon & No glucagon & $\begin{array}{l}\text { Aggressive } \\
\text { flushing }\end{array}$ & $\begin{array}{l}\text { No aggressive } \\
\text { flushing }\end{array}$ & Balloon & No balloon \\
\hline Total, $n(\%)$ & $58(50)$ & $58(50)$ & $6(5)$ & $110(95)$ & $28(24)$ & $88(76)$ \\
\hline Post-ERCP pancreatitis, $n$ & 4 & 5 & 2 & 7 & 4 & 5 \\
\hline No post-ERCP pancreatitis, $n$ & 54 & 53 & 4 & 103 & 24 & 83 \\
\hline \multicolumn{7}{|c|}{ Combination of intraoperative cholangiogram techniques and cases of post-endoscopic retrograde cholangiopancreatography pancreatitis } \\
\hline & \multicolumn{2}{|c|}{$\begin{array}{c}\text { Glucagon + Aggressive } \\
\text { flushing }\end{array}$} & on + Balloon & \multicolumn{2}{|c|}{$\begin{array}{c}\text { Aggressive flushing + } \\
\text { Balloon }\end{array}$} & $\begin{array}{l}\text { + Aggressive } \\
\text { + Balloon }\end{array}$ \\
\hline Total, $n$ & \multicolumn{2}{|l|}{5} & 11 & \multicolumn{2}{|l|}{3} & 2 \\
\hline Post-ERCP pancreatitis, $n$ & \multicolumn{2}{|l|}{2} & 2 & \multicolumn{2}{|l|}{2} & 2 \\
\hline No post-ERCP pancreatitis, $n$ & \multicolumn{2}{|l|}{3} & 9 & \multicolumn{2}{|l|}{1} & 0 \\
\hline
\end{tabular}

ERCP, endoscopic retrograde cholangiopancreatography.

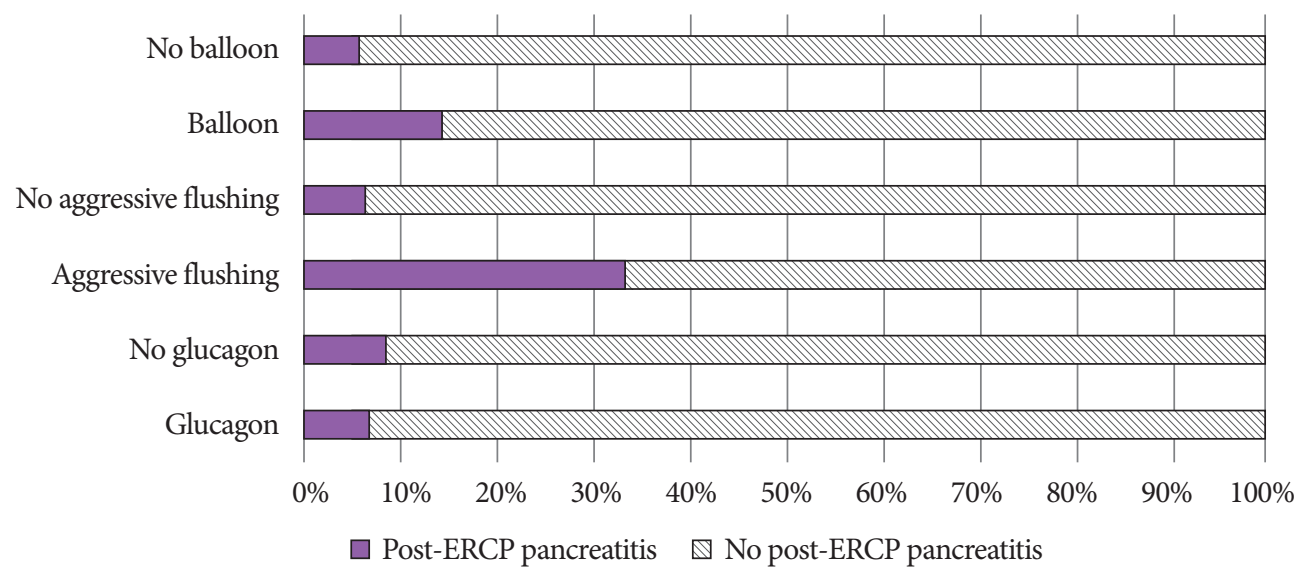

Fig. 1. Techniques applied during intraoperative cholangiogram and rates of post-endoscopic retrograde cholangiopancreatography (ERCP) pancreatitis. 
Table 3. Endoscopic Retrograde Cholangiopancreatography Techniques

\begin{tabular}{lccc}
\hline & Positive IOC $(\boldsymbol{n = 1 1 6})$ & No IOC $(\boldsymbol{n}=\mathbf{1 1 6})$ & $p$-value \\
\hline Any stent placed, $n(\%)$ & $32(27.6)$ & $53(45.7)$ & 0.004 \\
Pancreatic duct stent, $n(\%)$ & $13(11.2)$ & $14(12.1)$ & 0.23 \\
Rectal indomethacin, $n(\%)$ & $7(6.0)$ & $9(7.8)$ & 0.61 \\
Post-ERCP pancreatitis, $n(\%)$ & $9(7.8)$ & $3(2.6)$ & \\
\hline
\end{tabular}

ERCP, endoscopic retrograde cholangiopancreatography; IOC, intraoperative cholangiogram.

Table 4. Logistic Regression for Risk of Post-Endoscopic Retrograde Cholangiopancreatography Pancreatitis in Positive Intraoperative Cholangiogram

\begin{tabular}{lcc}
\hline & OR (95\% CI) & p-value \\
\hline Model 1: unadjusted & $3.17(0.835-12.02)$ & 0.09 \\
Model 2: adjusted for age, sex & $3.17(0.834-12.07)$ & 0.09 \\
\hline Model 3: adjusted for age, sex, stent placement, total bilirubin & $4.79(1.05-21.89)$ & 0.043 \\
Model 4: adjusted for age, sex, total bilirubin, alkaline phosphatase, stent placement & $4.69(1.02-21.50)$ & 0.047 \\
Model 5: adjusted for age, sex, total bilirubin, common bile duct diameter, stent placement & $6.27(1-39.27)$ & 0.05 \\
\hline Model 6: adjusted for age, sex, total bilirubin, alkaline phosphatase, stent placement, common & $6.27(0.99-39.81)$ & 0.051 \\
\hline bile duct diameter & $4.28(0.84-21.85)$ & 0.081 \\
\hline
\end{tabular}

$\mathrm{CI}$, confidence interval; $\mathrm{OR}$, odds ratio.

no clear trend of which technique could suggest PEP in the 9 patients; however, all techniques-glucagon injection, "aggressive" flushing, and balloon clearance devices-resulted in some patients developing PEP. Combinations of the IOC techniques also resulted in PEP, but the sample size was too small for statistical analysis.

The use of both common bile duct and PD stents was recorded (Table 3). Patients who did not undergo IOC were more likely to have any type of stent placed, corresponding to $46 \%$ of control patients, compared to only $28 \%$ in the positive IOC group. PD stents and rectal indomethacin were used similarly in both groups.

Of the 116 patients studied in each group, $7.8 \%$ developed PEP in the IOC group, compared to $2.6 \%$ of patients in the control group. Logistic regression analyses were performed to determine independent risk factors for PEP. The univariate model, multivariate adjusted models, and the final parsimonious multivariate model are presented in Table 4. After adjusting for age, total bilirubin levels, and stent placement (biliary and/or PD stents), the positive IOC group exhibited a significantly increased risk of PEP, nearly fivefold (odds ratio, 4.79; 95\% confidence interval, 1.05-21.89; $p<0.05$ ). Further adjustment for alkaline phosphatase levels and common bile duct diameter were also statistically significant, though com- mon bile duct diameter was not an independent predictor of PEP.

\section{DISCUSSION}

The burden of cholecystitis and choledocholithiasis is quite high in the general population. After the advent of IOC by Mirizzi in 1937, IOC has been used and recommended by many surgeons to delineate bile duct anatomy. ${ }^{1,11}$ There are also studies that support the use of IOC for suspected bile duct stones, with evidence of both success and cost-effectiveness. ${ }^{3,12}$ A study from 2018 showed an increasing incidence of bile duct injury with the decreasing use of IOC, suggesting that its routine use is recommended. ${ }^{2}$ However, guidelines are non-committal at best; the Society of American Gastrointestinal and Endoscopic Surgeons (SAGES) guideline in 2017 for IOC recommended the "liberal" use of IOC to prevent bile duct injury but stated that the evidence was "weak." There are also those who oppose its routine use, citing little significance in preventing bile duct injury against increased cost and operative time. ${ }^{14}$ Additionally, a systematic review of eight IOC clinical trials totaling 1,715 patients found no benefit in preventing retained bile duct stones. ${ }^{15}$ False positive rates have been reported in 
2016, at $31.5 \%$ for abnormal IOC with a normal ERCP. ${ }^{16}$ In the current study, though not all surgeons perform IOC at our center, they also will employ additional techniques for patients with a positive IOC, including glucagon injection, balloon clearance devices, and "aggressive" high-pressure flushing. The intention of this study was not to analyze the particular techniques employed during CCY with IOC. However, the evidence in this study associating IOC with an increased risk of PEP, suggests that further study of the impact of IOC techniques is warranted in the future to support a cautionary use of IOC in choledocholithiasis.

Though many risk factors have been studied, the pathophysiology of PEP is still not well-known. It is most widely accepted that the cause is multifactorial, involving chemical, thermal, mechanic, hydrostatic, enzymatic, allergic, and microbiological factors during ERCP. ${ }^{6,10,17}$ The hypothesis in the current study was that IOC causes stones to become impacted at the papilla, which can cause trauma to the papilla, papillary edema, and inadvertent PD injection. Risk factors were studied prospectively by Freeman et al. between $1995-1998 .{ }^{10}$ A few of the factors they found were female sex, normal bilirubin levels at the time of ERCP, difficult cannulation, and sphincter of Oddi dysfunction. ${ }^{10}$ Others have found that young age, a history of PEP, and a history of recurrent pancreatitis, in addition to procedural (sphincterotomy, manometry, etc.) and operator-related factors (e.g., lack of experience) can also increase the risk of PEP. ${ }^{17}$ In the current study, patients with a history of pancreatitis-PEP or gallstone pancreatitis-or prior sphincterotomy were excluded from both groups. Common bile duct size was not an independent predictor of PEP in our study, which has also been previously described. ${ }^{10}$ Unfortunately, all procedural factors could not be identified in this study, as our center does not have a system in place for standardized documentation of difficult cannulation. However, the logistic regression in this study adjusted for demographics, available pathologic factors, and procedural techniques, revealing an increased risk of PEP in patients with a positive IOC.

Given the results of the current study, we suggest that prophylaxis of PEP should be emphasized in patients with a positive IOC. Rectal administration of indomethacin, hydration with lactated Ringer's solution, and PD stents have all been shown to reduce the risk of $\mathrm{PEP}^{6,17-19}$ In this study, rectal indomethacin and PD stents were used in select patients (at the discretion of the endoscopist) in both groups to prevent PEP. The use of rectal indomethacin and PD stents was equally employed in both groups. The best method of prophylaxiswhether it is rectal indomethacin, a PD stent, aggressive hydration with lactated Ringer's solution, or a combination-needs to be further studied, especially in patients with a positive IOC.
There are several limitations to this study. As a tertiary care referral center, it is possible that more IOCs are performed at our center than, perhaps, in a community setting. However, likely due to the fact that there are no specific guidelines, the decision to perform IOC is left to the surgeon's preference. Another limitation of our study is the inability to control for all operator-dependent factors, as there are multiple surgeons and multiple endoscopists performing the procedures at our center. However, the presence of multiple providers, including trainees, also increases the generalizability of our results. In addition, we did not exclude false positive IOCs; thus, the actual rate may be much higher than reported in this study. We recognize this study was unable to capture those who potentially could develop post-IOC pancreatitis; it would be difficult to distinguish between post-operative pain and pancreatitis. Although the general low incidence of PEP constitutes an additional limitation, we counteracted the small sample size by using a case-control design to determine the risk of PEP and logistic regression to control for multiple variables and confounders.

In conclusion, the aim of this retrospective case-control study was to determine if there was an increased risk of PEP in patients with a positive IOC, which was defined as a persistent filling defect or lack of flow of contrast medium into the duodenum during CCY. These patients were compared to an age-sex matched cohort who had ERCP for choledocholithiasis without IOC or gallstone pancreatitis. To control for multiple variables, a parsimonious multivariable logistic regression model adjusted for age, sex, total bilirubin levels, and stent placement, revealed a nearly fivefold risk of PEP in patients with a positive IOC. Based on these findings, we suggest that patients should be selected carefully for IOC and be monitored after ERCP for PEP. The use of PEP prophylaxis in these patients, and the specific method, needs to be studied prospectively and will likely be helpful for the treatment of this subset of patients.

Conflicts of Interest

The authors have no potential conflicts of interest.

Funding

None.

Author Contributions

Conceptualization: Lalitha M. Sitaraman, David S. Lee

Data curation: LMS, Judith Kim

Formal analysis: LMS, Rita M. Knotts, Srihari Mahadev

Methodology: RMK, SM

Supervision: DSL

Writing-original draft: LMS

Writing-review\&editing: LMS, RMK, JK, SM, DSL 
ORCID

$\begin{array}{ll}\text { Lalitha M. Sitaraman: } & \text { https://orcid.org/0000-0003-4867-1236 } \\ \text { Rita M. Knotts: } & \text { https://orcid.org/0000-0002-3133-1772 } \\ \text { Judith Kim: } & \text { https://orcid.org/0000-0003-1859-0072 } \\ \text { Srihari Mahadev: } & \text { https://orcid.org/0000-0002-4123-3922 } \\ \text { David S. Lee: } & \text { https://orcid.org/0000-0002-0116-992X }\end{array}$

\section{REFERENCES}

1. Massarweh NN, Flum DR. Role of intraoperative cholangiography in avoiding bile duct injury. J Am Coll Surg 2007;204:656-664.

2. Altieri MS, Yang J, Obeid N, Zhu C, Talamini M, Pryor A. Increasing bile duct injury and decreasing utilization of intraoperative cholangiogram and common bile duct exploration over 14 years: an analysis of outcomes in New York State. Surg Endosc 2018;32:667-674.

3. Vecchio R, MacFadyen BV. Laparoscopic common bile duct exploration. Langenbecks Arch Surg 2002;387:45-54.

4. Lynn AP, Chong G, Thomson A. Endoscopic retrograde cholangiopancreatography in the treatment of intraoperatively demonstrated choledocholithiasis. Ann R Coll Surg Engl 2014;96:45-48.

5. Freeman ML, Nelson DB, Sherman S, et al. Complications of endoscopic biliary sphincterotomy. N Engl J Med 1996;335:909-918.

6. Tryliskyy Y, Bryce GJ. Post-ERCP pancreatitis: pathophysiology, early identification and risk stratification. Adv Clin Exp Med 2018;27:149154

7. Kochar B, Akshintala VS, Afghani E, et al. Incidence, severity, and mortality of post-ERCP pancreatitis: a systematic review by using randomized, controlled trials. Gastrointest Endosc 2015;81:143-149.e9.

8. Andriulli A, Loperfido S, Napolitano G, et al. Incidence rates of post-ERCP complications: a systematic survey of prospective studies. Am J Gastroenterol 2007;102:1781-1788.
9. Cotton PB, Garrow DA, Gallagher J, Romagnuolo J. Risk factors for complications after ERCP: a multivariate analysis of 11,497 procedures over 12 years. Gastrointest Endosc 2009;70:80-88.

10. Freeman ML, DiSario JA, Nelson DB, et al. Risk factors for post-ERCP pancreatitis: a prospective, multicenter study. Gastrointest Endosc 2001;54:425-434.

11. Mirizzi P. Operative cholangiography. Surg Gynecol Obstet 1937;65:702710

12. Orlando R 3rd, Russell JC. Managing gallbladder disease in a cost-effective manner. Surg Clin North Am 1996;76:117-128.

13. Hope WW, Fanelli R, Walsh DS, et al. SAGES clinical spotlight review: intraoperative cholangiography. Surg Endosc 2017;31:2007-2016.

14. Metcalfe MS, Ong T, Bruening MH, Iswariah H, Wemyss-Holden SA, Maddern GJ. Is laparoscopic intraoperative cholangiogram a matter of routine? Am J Surg 2004;187:475-481.

15. Ford JA, Soop M, Du J, Loveday BP, Rodgers M. Systematic review of intraoperative cholangiography in cholecystectomy. Br J Surg 2012;99:160167.

16. Bill JG, Kushnir VM, Mullady DK, et al. Evaluation of patients with abnormalities on intraoperative cholangiogram: time to abandon endoscopic retrograde cholangiopancreatography as the initial follow-up study. Frontline Gastroenterol 2016;7:105-109.

17. Parekh PJ, Majithia R, Sikka SK, Baron TH. The "Scope" of post-ERCP pancreatitis. Mayo Clin Proc 2017;92:434-448.

18. Mok SRS, Ho HC, Shah P, Patel M, Gaughan JP, Elfant AB. Lactated Ringer's solution in combination with rectal indomethacin for prevention of post-ERCP pancreatitis and readmission: a prospective randomized, double-blinded, placebo-controlled trial. Gastrointest Endosc 2017;85:1005-1013

19. Mazaki T, Mado K, Masuda H, Shiono M. Prophylactic pancreatic stent placement and post-ERCP pancreatitis: an updated meta-analysis. J Gastroenterol 2014;49:343-355. 